Pacific Journal of Mathematics

CLOSED ULTRAFILTER AND REALCOMPACTNESS 


\title{
CLOSED ULTRAFILTERS AND REALCOMPACTNESS
}

\author{
TAKESI ISIWATA
}

\begin{abstract}
We introduce some conditions which are closely related to closed ultrafilters and establish interconnections among these conditions and characterize realcompactness, almost realcompactness, $c$-realcompactness and weak- $c b^{*}$-ness $\cdots$.
\end{abstract}

We introduce some conditions which are closely related to closed ultrafilters and establish interconnections among these conditions and characterize realcompactness, alomost realcompactness, $c$-realcompactness and weak-c $c b^{*}$-ness, $\cdots$.

Throughout this paper, by a space we mean a completely regular Hausdorff space and all functions are continuous and we assume familiarity with [3] whose notation and terminology will be used throughout. For a given space $X$, we denote by $\beta X$ (or $\cup X$ ) the Stone-Čech compactification (or realcompactification) of $X$. In $\S 1$, we give definitions and preliminaries and introduce some conditions which are closely related to closed ultrafilters. In $\S 2$, we establish interconnections among conditions introduced in $\S 1$. In $\S 3$, we characterize realcompactness, almost realcompactness $c$-realcompactness and weak$c b^{*}$-ness and give some examples in $\S 4$.

Notations and terminologies. $N=$ the set of positive integers, $\mathrm{nbd}=$ neighborhood, $\omega=$ the first countable ordinal, $\Omega=$ the first uncountable ordinal, $C(X)=$ the ring of all continuous functions on $X, Z(f)=$ the zero set of $f \in C(X)$ where we assume $0 \leqq f \leqq 1$, $Z(X)=$ the set of all zero sets, $X^{*}=\beta X-X$. $\mathscr{F}(\mathscr{U}$ or $\mathscr{R}$ resp.) $=$ a free closed (open or regular closed resp.) ultrafilter. $\mathscr{F}^{p}\left(\mathscr{Z}^{p}\right)=$ a free closed $(Z)$ ultrafilter converging to $p \in X^{*}$. $\widetilde{\mho}=$ the set of all $\mathscr{F}$ (similarly define $\mathscr{U}^{p}, \mathfrak{H}$ and $\Re$ resp.), $\operatorname{cl} \mathscr{C}=\{\operatorname{cl} U ; U \in \mathscr{W}\}$ and $\left\{F_{n}\right\}_{c 1} \downarrow\left(\left\{F_{n}\right\}_{c 1} \downarrow \varnothing\right)=$ a decreasing sequence of closed sets (with the empty intersection). Similarly we define $\left\{R_{n}\right\}_{r c} \downarrow$ and $\left\{Z_{n}\right\}_{z e} \downarrow \cdots$ where " $r c$ " and " $z e$ " denote " $R_{n}$ is a regular closed set" and " $Z_{n}$ is a zero set" respectively.

1. Definitions and preliminaries. A family $\mathscr{A}$ of subsets of $X$ is said to be stable if for any $f \in C(X)$ there is $A \in \mathscr{A}$ such that $f \mid A$ is bounded. Mandelker ([10], Th. 5.1) has proved that $X$ is realcompact iff any stable closed family $\mathscr{A}$ with the finite intersection property has non-empty intersection and Hardy and Woods ([4], Lemma 2.6) have obtained that $\mathscr{R}$ is stable iff there is $p \in \cup X-X$ and $\mathscr{R} \rightarrow p$. We say that $\mathscr{C}$ or $\mathscr{R}$ has $C I P$ if $\cap \operatorname{cl} A_{n} \neq \varnothing$ for any 
$A_{n} \in \mathscr{U}$ or $\mathscr{R}$ respectively.

1.1. (1) $\mathscr{A} \in \widetilde{F}($ or $\mathfrak{i})$ is stable iff . $\mathscr{A}$ converges to some point of $\cup X-X$.

(2) $X$ is realcompact iff no $\mathscr{F}$ is stable.

(3) For $p \in \beta X-\cup X$, no prime closed (resp. open) filter $\mathscr{A}$ converging to $p$ has CIP.

Proof. (1) From the same method used in the proof of Lemma 2.6 in [4]. (2) From (1). (3) Let $p \in \beta X-v X$. Then there is $f \in C(\beta X)$ with $p \in Z(f) \subset \beta X-v X$. Since $\mathscr{A}$ is prime, $\mathscr{A} \ni F_{n}=$ $\{x \in X ; f(x) \leqq 1 / n\}\left(\right.$ resp. $\left.U_{n}=\{x \in X ; f(x)<1 / n\}\right)$ and hence $\mathscr{A}$ does not have CIP.

The following are well known (e.g., p. 649 line 4 in [4]).

1.2. (1) $\mathfrak{U} \ni \mathscr{U} \Rightarrow \mathscr{R}=\operatorname{cl} \mathscr{U} \in \Re$. If $\mathscr{U}$ has $C I P$, so has $\mathscr{R}$.

(2) $\Re \ni \mathscr{R} \Rightarrow \mathscr{U}(R)=\{U$; int $R \subset U$ for some $R \in \mathscr{R}$ and $U$ is open $\} \in \mathfrak{U}$. If $\mathscr{R}$ has $C I P$, so has $\mathscr{U}(\mathscr{R})$.

(3) $\mathfrak{u} \ni \mathscr{W} \Rightarrow \mathscr{U}(\operatorname{cl} \mathscr{U})=\mathscr{U}$.

(4) $\Re \ni \mathscr{R} \Rightarrow \operatorname{cl}(\mathscr{C}(\mathscr{R}))=\mathscr{R}$.

1.3. We will divide $X^{*}$, especially $v X-X$, into three domains.

$$
\mathfrak{F}(0)=\left\{p \in X^{*} ; \text { any } \mathscr{F}^{p} \text { has } C I P\right\} \text {. }
$$

$\mathfrak{F}(0, \triangle)=\left\{p \in X^{*}\right.$; there are $\mathscr{F}_{1}^{p}$ with $C I P$ and $\mathscr{F}_{2}{ }^{p}$ without $\left.C I P\right\}$. $\mathfrak{F}(\triangle)=\left\{p \in X^{*} ;\right.$ no $\mathscr{F}^{p}$ has $\left.C I P\right\}$.

$\mathfrak{F}(\nu, \triangle)=\mathfrak{F}(\triangle) \cap(u X-X)$.

Similarly we define $\mathfrak{H}(0), \mathfrak{u}(0, \triangle), \mathfrak{M}(\triangle), \mathfrak{H}(v, \triangle), \mathfrak{R}(0), \mathfrak{R}(0, \Delta)$, $\mathfrak{R}(\triangle)$ and $\mathfrak{R}(\nu, \triangle)$. From 1.1(3) and 1.2 we have $\mathfrak{H}(0)=\mathfrak{R}(0), \mathfrak{u}(0, \triangle)=$ $\mathfrak{R}(0, \triangle), \mathfrak{H}(\triangle)=\mathfrak{R}(\triangle)$ and $\beta X-v X \subset \mathfrak{H}(\triangle)$.

1.4. As generalization of realcompactness, almost realcompactness [1], $c$-realcompactness [2] and $a$-realcompactness [2] are introduced. $X$ is said to be almost realcompact if any open ultrafilter $\mathscr{C}$ with $C I P$ is fixed, that is, $\cap$ cl $\mathscr{C} \neq \varnothing[1] . \quad X$ is a-realcompact (= closedcomplete) if any closed ultrafilter $\mathscr{F}$ with $C I P$ is fixed [2]. We say that $X$ is c-realcompact if for each point $p \in \beta X-X$, there exists $\left\{R_{n}\right\}_{r c} \downarrow \varnothing$ with $p \in \cap \mathrm{cl}_{\beta X} R_{n}$ [5]. From 1.2 and the definition we have

(1) $X$ is almost realcompact iff $\mathfrak{u}(0) \cup \mathfrak{u}(0, \triangle)=\varnothing$.

(2) $X$ is c-realcompact iff $\mathfrak{u}(0)=\varnothing$.

(3) $X$ is a-realcompact iff $\mathfrak{\xi}(0) \cup \mathfrak{\Im}(0, \triangle)=\varnothing$. 
From these results and 1.2 , it is natural to introduce the notion of wa-realcompactness, that is, we say that $X$ is wa-realcompact if $\mathfrak{F}(0)=\varnothing$. Since $\mathfrak{F}(0, \Delta)=\varnothing$ for a normal space, a normal $w a$ realcompact space is $a$-realcompact. If $X$ is either $c$-realcompact or $a$-realcompact, then $X$ is $w a$-realcompact (cf. 2.1(1) below). The converse is not necessarily true (see 4.1 and 4.2 below). As another generalization of $c b$ (weak-cb)-ness, we introduced the notion of $c b^{*}$ (weak-c $c b^{*}$ ) spaces [8]. $X$ is said to be $c b^{*}$ (weak-c $b^{*}$ ) if $\cap \mathrm{cl}_{v X} F_{n}=$ $\varnothing\left(\cap \mathrm{cl}_{\nu_{X}} R_{n}=\varnothing\right)$ for any $\left\{F_{n}\right\}_{\mathrm{cl}} \downarrow \varnothing\left(\left\{R_{n}\right\}_{r c} \downarrow \varnothing\right)$. In [8] we proved that $X$ is $c b^{*}$ iff any perfect map onto $X$ is hyper-real. The following are easily seen by $1.1(3), 1.3$ and the definitions.

(4) $X$ is weak-cb* iff $\mathfrak{H}(\nu, \triangle) \cup \mathfrak{H}(0, \triangle)=\varnothing$.

(5) $X$ is $c b^{*}$ iff $\mathfrak{F}(\cup, \triangle) \cup \mathfrak{F}(0, \triangle)=\varnothing$.

(6) $X$ is realcompact iff $X$ is wa-realcompact and $c b^{*}$.

( 7 ) $X$ is countably compact iff $\mathfrak{F}(0, \triangle) \cup \mathfrak{F}(\triangle)=\varnothing$.

(8) $X$ is pseudocompact iff $\mathfrak{H}(0, \triangle) \cup \mathfrak{H}(\triangle)=\varnothing$.

DEFINITION 1.5. $\widetilde{F}$ is said to be countably paracompact $(=c p)$ (weakly countably paracompact $(=w c p)$ ) if for any $\left\{F_{n} \in \mathscr{F}\right\} \downarrow \varnothing$ there are $\left\{E_{n} \in F\right\} \downarrow \varnothing$ and $\left\{U_{n}\right\}_{\text {open }} \downarrow$ such that $E_{n} \subset F_{n}, E_{n} \subset U_{n}\left(E_{n} \subset\right.$ $\left.\operatorname{cl} U_{n}\right)$ and $\cap \operatorname{cl} U_{n}=\varnothing$. Obviously we have

(1) If $\mathscr{F}$ is either unstable or has CIP, then it is cp.

(2) $A$ cp $\mathscr{F}$ is wcp.

Definition 1.6. For $\mathscr{F}$ (or $\mathscr{U}$ ), we denote by $\mathfrak{M}(\mathscr{F})$ (or $\mathfrak{F}(\mathscr{U})$ ) the set of open (or closed) ultrafilters containing $\{U ; U \supset F$ for some $F \in \mathscr{F}$ (or cl $\mathscr{C})\}$. In the sequel, " $\mathfrak{F}(\mathscr{C}) \rightarrow p$ " means "any $\mathscr{F} \in \mathfrak{F}(\mathscr{C})$

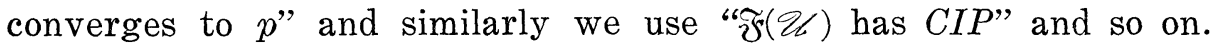
Then we have

(1) $\mathscr{F} \rightarrow p$ implies $\mathfrak{H}(\mathscr{F}) \rightarrow p$ and $\operatorname{cl} \mathfrak{H}(\mathscr{F}) \subset \mathscr{F}$. If $\mathscr{F}$ has CIP, so has $\mathfrak{U}(\mathscr{F})$.

(2) $\mathscr{U} \rightarrow p$ implies $\mathfrak{F}(\mathscr{U}) \rightarrow p$. If $\mathscr{C}$ does not have CIP, then any $\mathscr{F} \in \mathfrak{F}(\mathscr{Q})$ is wcp but does not have CIP.

(3) For a given $\mathscr{U}, \mathscr{U} \in \mathfrak{H}(\mathscr{F})$ for any $\mathscr{F} \in \mathfrak{F}(\mathscr{C})$.

Proof. (1) and (2) are obvious. (3) Let $\mathscr{F} \in \mathfrak{F}(\mathscr{C})$ and let $W$ be an open set containing some $F \in \mathscr{F}$. If $W \notin \mathscr{Q}$, then there is $U \in$ $\mathscr{U}$ with $U \cap W=\varnothing$, so $\mathscr{F} \ni F \cap \operatorname{cl} U=\varnothing$, a contradiction. Thus $W \in \mathscr{C}$ and hence $\mathscr{C} \in \mathfrak{H}(\mathscr{F})$.

DeFinition 1.7. We consider the following conditions. $(\alpha)((\beta))$ For any $p \in X^{*}$, there is a wcp $(c p) \mathscr{F}^{p}$. $(s \alpha)((s \beta))$ For any $p \in X^{*}$, any $\mathscr{F}^{p}$ is wcp $(c p)$.

The following (1) and (2) follows from the definitions and the 
fact that if $X$ is normal, then the closed ultrafilter converging to $p \in X^{*}$ is only one.

(1) $(s \beta) \Rightarrow(\beta) \Rightarrow(\alpha)$ and $(s \beta) \Rightarrow(s \alpha) \Rightarrow(\alpha)$.

(2) If $X$ is normal, then $(\beta) \Rightarrow(s \beta)$ and $(\alpha) \Rightarrow(s \alpha)$.

From $\beta X-v X \subset \mathfrak{U}(\triangle), 1.1(3), 1.4(5), 1.6(1)$ and the result that $X$ is countably paracompact iff for any $\left\{F_{n}\right\}_{\mathrm{c} 1} \downarrow \varnothing$, there is $\left\{U_{n} ; F_{n} \subset U_{n}\right\}_{\text {open }} \downarrow$ $\varnothing$ with $\cap \operatorname{cl} U_{n}=\varnothing[7]$, we have $(s \beta)$.

(3) If $X$ is either countably paracompact or $c b^{*}$ then $X$ has

There is a normal space which has neither $(\alpha)$ nor $(\beta)$ (see 4.1 below).

1.8. As one of the nice property of the zero sets, it is known that a $Z$-ultrafilter $\mathscr{Z}$ has $C I P$ iff $\mathscr{Z}$ contains a prime $Z$-filter with CIP. Unfortunately this is not necessarily true for closed ultrafilters. N. Dykes [1] has, however, proved that if $X$ is a $c b$-space, $F$ has CIP iff $\mathscr{F}$ contains a prime filter with CIP. In the following we treat the related problem above. We consider the following conditions.

$(C P C)$ If $\mathscr{F}$ contains a prime closed filter with CIP, then $\mathscr{F}$ has CIP.

$(O P C)$ If $\mathscr{A}$ is a prime open filter with CIP and $\mathrm{cl} \mathscr{A} \subset \mathscr{F}$, then $\mathscr{F}$ has CIP.

(OPO) If $\mathscr{C}$ contains a prime open filter with CIP, then $\mathscr{U}$ has CIP.

(WOPC) If $\mathscr{C}$ has CIP, then $\mathfrak{(}(\mathscr{C})$ has CIP.

$(Z C)$ If $Z(X) \cap \mathscr{F}=\mathscr{Z}^{p}$ for $p \in \cup X-X$, then $\mathscr{F}$ has CIP (cf. $2.2(3))$.

Notice that (1) $\mathscr{Z}^{p}$ has $C I P$ iff $p \in \cup X-X$ and (2) if $\mathscr{F}$ has $C I P$, then $\mathscr{F} \cap Z(X)=\mathscr{Z}^{p}$ for some $p \in \cup X-X$ by 2.2(3) below. From 1.1(3), 1.4(4.5) and the definition, we have the following implications:

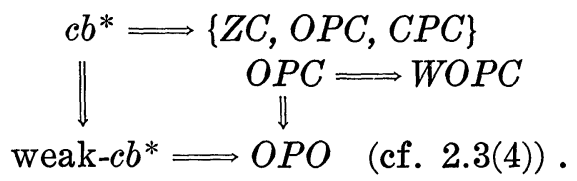

There is a weak-cb normal space without $Z C$ (see, 4.1 below).

2. Interconnections among conditions introduced in $\S 1$.

2.1. (1) $\mathfrak{F}(0) \subset \mathfrak{H}(0)$ and $\mathfrak{H}(\triangle) \subset \mathfrak{F}(\triangle)$.

(2) Let $p \in X^{*}$. Then there is $\mathscr{Q}^{p}$ without CIP iff there is wcp $\mathscr{F}^{p}$ without CIP. Equivalently let $p \in X^{*}$, then $p \in \mathfrak{H}(0)$ iff no $\mathscr{F}^{p}$ without CIP is wcp. 
(3) $p \in \mathfrak{H}(0) \cap \mathfrak{F}(\triangle)$ iff no $\mathscr{F}^{p}$ has $C I P$ and no $\mathscr{F}^{p}$ is wcp.

(4) $\quad p \in \mathfrak{U}(0) \cap \mathfrak{F}(0, \triangle)$ iff there are $\mathscr{F}_{1}^{p}$ with CIP and $\mathscr{F}_{2}^{p}$ without CIP and no $\mathscr{F}^{p}$ without CIP is wcp.

(5) $\quad p \in \mathfrak{F}(0, \triangle) \cap \mathfrak{H}(0, \triangle)$ iff there are $\mathscr{F}_{1}^{p}$ with CIP and a wep $\mathscr{F}_{2}{ }^{p}$ without CIP.

Proof. (1) Let $p \in \mathfrak{F}(0)$ and $\mathscr{U} \rightarrow p$. By 1.6(2), $\mathfrak{F}(\mathscr{U}) \rightarrow p$, cl $\mathscr{U} \subset$ $\mathscr{F}$ for each $\mathscr{F} \in \mathfrak{F}(\mathscr{U})$ and $\mathfrak{F}(\mathscr{U})$ has $C I P$, and hence $\mathscr{U}$ has $C I P$, so $p \in \mathfrak{U}(0)$. Now suppose that $p \in \mathfrak{H}(\triangle)$ and $\mathscr{F} \rightarrow p$. Then $\mathfrak{U}(\mathscr{F})$ does not have CIP. By 1.6(1) $\mathscr{F}$ does not have $C I P$, so $p \in \mathfrak{F}(\triangle)$.

$(2) \Rightarrow$ From 1.6. (2) $\Longleftarrow$. By the assumption, there is an open set $U_{n}(n \in N), \operatorname{cl} U_{n} \in \mathscr{F}$ with $\cap \operatorname{cl} U_{n}=\varnothing$. Let $N(p)$ be the open nbd system of $p$ in $\beta X$ and let $\mathscr{C} \supset\left\{U_{n} \cap V ; n \in N, V \in N(p)\right\}$. Obviously $\mathscr{Q}$ converges to $p$ but does not have $C I P$.

$(3) \Longrightarrow$ Obvious. $\sqsubset$. From (2).

$(4) \Rightarrow$ From $p \in \mathfrak{F}(0, \triangle)$ and $(2)$. $\Rightarrow$. From (2) and the assumption.

$(5) \Rightarrow$ Since $p \in \mathfrak{F}(0, \triangle)$, there is $\mathscr{F}_{1}{ }^{p}$ with CIP. By (2), $p \in$ $\mathfrak{u}(0, \triangle)$ implies that there is a wcp $\mathscr{F}_{2}^{p}$ without $C I P . \Longleftarrow$. By the assumption, we have $p \in \mathfrak{F}(0, \triangle)$ and $p \in \mathfrak{M}(0, \triangle)$ by (1) and (2).

Lemma 2.2. (1) If $\mathscr{U}^{p}$ has CIP, then $\mathscr{A}=\{F ; F$ contains some $U \in \mathscr{U}$ and $F$ is closed $\}$ is a prime closed filter with CIP and cl $\mathscr{U}^{p} \subset$ $\mathscr{F}$ whenever $\mathscr{A} \subset \mathscr{F}$.

(2) Let $X$ is normal and let $p \in \cup X-X$. Then $\mathscr{A}=\{U ; U$ contains some $Z \in \mathscr{L}^{p}$ and $U$ is open $\}$ is a prime open filter with CIP. If $\mathscr{U} \rightarrow p$, then $\mathscr{A} \subset \mathscr{U}$.

(3) If $\mathscr{F}$ has CIP, then $Z(X) \cap \mathscr{F}=\mathscr{Z}^{p}$ for some $p \in$ $v X-X$.

Proof. (1) It is sufficient to show that $\mathscr{A}$ is prime. Let $E$ and $F$ be closed, $E \cup F \in \mathscr{A}$ and $E \notin \mathscr{A}$. Then $E \cup F \supset \operatorname{int}(E \cup F)=$ $A \in \mathscr{U}$ and int $E \notin \mathscr{C}$. Since $\mathscr{U}$ is a ultrafilter and int $E \cup(X-E)$ is dense in $X$, we have $X-E \in \mathscr{U}$, so $A \cap(X-E) \in \mathscr{W}$. Since $A \cap$ $(X-E) \subset \operatorname{int} F$, we have $F \in \mathscr{A}$. The latter part is obvious.

(2) It suffices to show that $\mathscr{A}$ is prime. Suppose not; Let $W$ and $V$ be open and let $W \cup V \in \mathscr{A}, W \notin \mathscr{A}$ and $V \notin \mathscr{A}$, then there is $Z \in \mathscr{Z}^{p}$ such that $Z \subset W \cup V$. As $W \notin \mathscr{A}$ and $V \notin \mathscr{A}, Z-W \neq \varnothing$ and $Z-V \neq \varnothing$. Since $X$ is normal, there are zero sets $Z_{1}$ and $Z_{2}$ such that $X-V \subset X-Z_{1}, Z-W \subset X-Z_{2}$ and $\left(X-Z_{1}\right) \cap\left(X-Z_{2}\right)=$ $\varnothing$. Thus $Z_{1} \subset V$, so as $V \notin \mathscr{A}, Z_{1} \notin \mathscr{Z}^{p}$. Thus $Z_{2} \in \mathscr{Z}^{p}$. Similarly, there are zero sets $Z_{3}$ and $Z_{4}$ such that $X-W \subset X-Z_{3}, Z-V \subset$ $X-Z_{4}$ and $\left(X-Z_{3}\right) \cap\left(X-Z_{4}\right)=\varnothing$. As above, $Z_{4} \in \mathscr{Z}^{p}$. Thus $Z \cap$ $Z_{2} \cap Z_{4} \in \mathscr{L}^{p}$. But $Z \cap Z_{2} \cap Z_{4} \subset W \cap V$, so $W$ and $V \in \mathscr{A}$, which is a contradiction. Hence $\mathscr{A}$ is prime. 
(3) In general, it is evident that $\mathscr{A} \cap Z(X)$ is a prime $Z$-filter for a prime closed filter $\mathscr{A}$. Suppose that there is $Z \in Z(X)$ which intersects each member of $Z(X) \cap \mathscr{F}$, but $Z \notin \mathscr{F}$. Let $Z=Z(f), f \in$ $C(X), A_{n}=\{x ; f(x) \geqq 1 / n\}$ and $B_{n}=\{x: f(x) \leqq 1 / n\}$. Since $A_{n} \cup B_{n}=$ $X, \mathscr{F}$ contains $A_{n}$ or $B_{n}$. If $B_{n} \in \mathscr{F}$ for infinitely many $n$, then $Z=\cap B_{n} \in \mathscr{F}$, a contradiction. Thus $A_{n} \in \mathscr{F}$ for some $n$, and hence $A_{n} \in \mathscr{F} \cap Z(X)$, a contradiction, so $\mathscr{F} \cap Z(X)=Z^{p}$ for some $p \in X^{*}$. By 1.1(3) we have $p \in \cup X-X$.

Notice that the assumption $C I P$ in $2.2(3)$ is essential as is shown in 4.3 below.

2.3. (1) $X$ has $(\alpha)$ iff $\mathfrak{u}(0) \cap \mathfrak{F}(\triangle)=\varnothing$.

(2) $X$ has $(s \alpha)$, then $\mathfrak{u}(0)=\mathfrak{F}(0)$.

(3) $X$ has WOPC iff $\mathscr{F}$ does not have CIP, neither has $\mathfrak{u}(\mathscr{F})$.

(4) If $X$ has CPC, then $X$ has WOPC.

(5) If $X$ has $(s \beta)$, then $X$ has WOPC.

(6) If $X$ has $W O P C$, then $\mathfrak{\Im}(0)=\mathfrak{U}(0), \mathfrak{\Im}(0, \triangle)=\mathfrak{U}(0, \triangle)$ and $\mathscr{F}(\triangle)=\mathfrak{H}(\triangle)$.

(7) If $X$ is normal, then $X$ has WOPC iff $\mathfrak{F}(0)=\mathfrak{u}(0)$ and $\mathfrak{F}(\triangle)=\mathfrak{H}(\triangle)$.

(8) If $X$ has $Z C$, then $\mathfrak{F}(\nu, \triangle)=\varnothing$ (and hence $\mathfrak{H}(\nu, \triangle)=\varnothing)$.

(9) If $\mathscr{F} \cap Z(X)$ has $C I P$, so has $\mathscr{Z}^{p}$ (and hence $p \in \cup X-X$ ).

Proof. $\quad(1) \Rightarrow$ From $2.1(3) . \Longleftarrow$. Since any $\mathscr{F}$ with $C I P$ is $w c p$, we consider only a point $p \in \mathfrak{F}(\triangle)$. By the assumption, $p \notin \mathfrak{U}(0)$, so there is $\mathscr{U}^{p}$ without $C I P$. Thus there is a $w c p \mathscr{F}^{p}$ without $C I P$ by $2.1(2)$.

(2) $(s \alpha)$ implies $\mathfrak{u}(0) \cap \mathfrak{F}(0, \triangle)=\varnothing$ by $2.1(4)$, so $\mathfrak{U}(0)=\mathfrak{F}(0)$ by (1) and 2.1(1).

$(3) \Longrightarrow$ Suppose that $\mathscr{F}_{0}$ does not have $C I P$ but some $\mathscr{L}_{0} \in \mathfrak{U}\left(\mathscr{F}_{0}\right)$ has CIP. Since $X$ has $W O P C, \mathfrak{\mho}\left(\mathscr{U}_{0}\right)$ has CIP. On the other hand, cl $\mathscr{U}_{0} \subset \mathscr{F}_{0}$, so $\mathscr{F}_{0} \in \widetilde{F}\left(\mathscr{U}_{0}\right)$ which shows that $\mathscr{F}_{0}$ has $C I P$, a contradiction. $=$. Suppose that $\mathscr{U}_{0}$ has $C I P$ but some $\mathscr{F}_{0} \in \mathfrak{F}\left(\mathscr{U}_{0}\right)$ does not have CIP. By 1.6(3), $\mathscr{W}_{0} \in \mathfrak{U}\left(\mathscr{F}_{0}\right)$ and $\mathfrak{U}\left(\mathscr{F}_{0}\right)$ does not have $C I P$, a contradiction.

(4) Take $\mathscr{U}^{p}$ with $C I P$ and let $\mathscr{F} \supset \mathrm{cl} \mathscr{U}^{p}$. Then $\mathscr{F}$ contains a prime closed filter $\mathscr{A}$ described in $2.2(1)$, and hence $\mathscr{F}$ has $C I P$. Thus $\mathfrak{F}\left(\mathscr{U}^{p}\right)$ has $C I P$.

(5) Take $\mathscr{F}=\mathscr{F}^{p}$ without $C I P$ and let $\mathscr{U} \in \mathfrak{H}(\mathscr{F})$. Since $U \supset\{W ; W$ is open and $W \supset F$ for some $F \in \mathscr{F})$ and $\mathscr{F}$ is $c p$, there is $\left\{W_{n} ; W_{n} \in \mathscr{U}\right\}$ with $\cap \operatorname{cl} W_{n}=\varnothing$. Thus $\mathfrak{u}(\mathscr{F})$ does not have $C I P$ and $X$ has $W O P C$ by (3).

(6) Let $p \in \mathfrak{U}(0, \triangle) \cup \mathfrak{H}(0)$ and take $\mathscr{U}^{p}$ with $C I P$. By the assumption, $\mathfrak{F}\left(\mathscr{C}^{p}\right)$ has $C I P$, so $p \notin \mathfrak{F}(\triangle)$ which shows $\mathfrak{F}(\triangle)=\mathfrak{H}(\triangle)$ 
by 2.1(1). Let $p \in \mathfrak{F}(0, \triangle) \cup \mathfrak{U}(0)$ and take $\mathscr{F}^{p}$ without $C I P$. Then $\mathfrak{H}\left(\mathscr{F}^{p}\right)$ does not have CIP by (3), a contradiction. This shows $\mathfrak{U}(0)=\mathfrak{F}(0)$.

$(7) \Rightarrow$ From $(6)$ and $\mathfrak{F}(0, \triangle)=\varnothing . \Longleftarrow$ Since $X$ is normal, $\mathfrak{F}(0, \triangle)=\varnothing$. Take $\mathscr{F}^{p}$ without $C I P$. Then $p \in \mathfrak{F}(\triangle)=\mathfrak{H}(\triangle)$, and hence $\mathfrak{i}(\mathscr{F})$ does not have $C I P$, so $X$ has WOPC by (3).

(8) Let $p \in \mathfrak{F}(v, \triangle)$ and take $\mathscr{F} \supset \mathscr{F}^{p}$. Since $X$ has $Z C$, $\mathscr{F}$ has $C I P$, a contradiction.

(9) Suppose that $\mathscr{Z}^{p}$ does not have CIP. Then $p \in \beta X-\nu X$ and hence $\mathscr{L}^{p}$ contains $F_{n}$ described in 1.1(3). Since $F_{n} \in \mathscr{F}^{p}, \mathscr{F}^{p} \cap Z(X)$ does not have $C I P$, a contradiction.

By $1.4(1,2)$ and $2.3(1,6)$, we have the following implications.

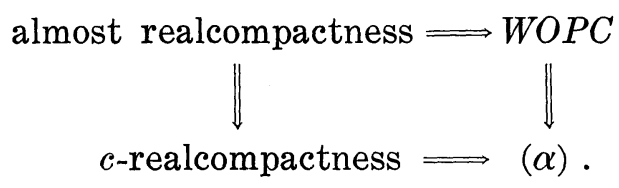

3. Characterizations of spaces of by means closed ultrafilters.

THEOREM 3.1. (1) $X$ is weak-c $b^{*}$ iff no $\mathscr{F}^{p}$ without CIP is wep for $p \in X^{*}$.

(2) A normal space $X$ is weak-cb* iff $X$ has OPO.

(3) $X$ is $c b^{*}$ iff $X$ is weak-c $b^{*}$ and has WOPC.

(4) The following are equivalent for a normal space $X$ : (i) $X$ is $c b^{*}$ (ii) $X$ has OPC. (iii) Any stable $\mathscr{F}$ is cp. (iv) $X$ has $Z C$.

(5) A pseudocompact space $X$ is countably compact iff $X$ has WOPC.

Proof. (1) From 2.1(2).

(2) Since a weak-cb* space has $O P O$ by the diagram of 1.8 , it suffices to show the converse. Let $p \in(v X-X)-\mathfrak{U}(0)$ and take any $\mathscr{U}^{p}$ without CIP. $X$ being normal, there is a prime open filter $\mathscr{A}$ with $C I P$ and $\mathscr{A} \subset \mathscr{U}^{p}$ by $2.2(2)$, so $\mathscr{U}^{p}$ has $C I P$ by the assumption which is a contradiction.

(3) A $c b^{*}$ space is weak-cb and has WOPC by 1.8. Conversely suppose that $X$ is weak- $c b^{*}$ and has WOPC. $X$ being weak-c $c b^{*}, v X-$ $X=\mathfrak{H}(0)$, so $\mathfrak{F}(0)=\mathfrak{H}(0)$ by $2.3(6)$, and hence $X$ is $c b^{*}$.

(4) $\quad$ (i) $\Rightarrow$ (ii) From the diagram of 1.8 .

(ii) $\Rightarrow$ (iii) Let $\mathscr{F}$ be stable. Then $\mathscr{F} \rightarrow p$ for some $p \in \cup X-X$ by 1.1(1). By the diagram of 1.8 and $2.3(7)$, we have $\mathfrak{F}(0)=\mathfrak{U}(0)$ and $\mathfrak{F}(v, \triangle)=\mathfrak{H}(v, \triangle)$. Since $X$ is normal, $\mathscr{\varkappa}^{p} \subset \mathscr{F}^{p}$, and hence $\mathscr{F}$ contains $\mathscr{A}$ described in $2.2(2)$, so $\mathscr{F}$ has $C I P$ by $O P C$ which shows that $\mathscr{F}$ is $c p$ by $1.5(1)$. 
(iii) $\Rightarrow$ (iv) Since $X$ is normal, any $\mathscr{F}^{p}$ contains $\mathscr{Z}^{p}$. We suppose that there are $p \in v X-X$ and $\mathscr{F}=\mathscr{F}^{p}$ without CIP. Then there is $\left\{U_{n}\right\}_{\text {open }} \downarrow$ such that $F_{n} \subset U_{n}$ for some $F_{n} \in \mathscr{F}$ and $\cap \operatorname{cl} U_{n}=\varnothing . \quad X$ being normal, it is easily seen that there is $Z_{n} \in Z(X)$ with $F_{n} \subset$ $Z_{n} \subset U_{n}$, so $\cap Z_{n}=\varnothing$, a contradiction.

(iv) $\Rightarrow$ (i) For $p \in \cup X-X$, $\mathscr{Z}^{p}$ has $C I P$ and hence any $\mathscr{F}$ containing $\mathscr{Z}^{p}$ has $C I P$ by $Z C$. Thus $\mathfrak{F}(\triangle)=\varnothing$. On the other hand, $X$ being normal $\mathfrak{F}(0, \triangle)=\varnothing$, and hence $\cup X-X=\mathscr{F}(0)$, so $X$ is $c b^{*}$.

(5) If $X$ is countably compact, then $X$ is $c b^{*}$ [8], so $X$ has $W O P C$ by (3). Conversely, if $X$ has $W O P C$, then $X$ is $c b^{*}$ by (3) because a pseudocompact space is weak-eb*. Thus $X$ is countably compact [8].

$X$ is said to be almost normal ((v)-almost normal) if a closed subset $F$ disjoint from a regular closed subset $E$, then $\operatorname{cl}_{\beta X} F \cap \mathrm{cl}_{\beta X} E=$ $\varnothing\left(\mathrm{cl}_{v X} F \cap \mathrm{cl}_{u X} E=\varnothing\right)$. It is obvious that $X$ is almost normal ((v)almost normal) iff $\mathrm{cl} \mathscr{U}^{p} \subset \mathscr{F}^{p}$ for each $\mathscr{F}^{p}$ and each $\mathscr{U}^{p}$ for $p \in$ $X^{*}(p \in \cup X-X)$.

3.2. Let $X$ be (v)-almost normal. Then we have

(1) $\mathfrak{\Im}(0, \Delta) \cap \mathfrak{u}(0, \Delta)=\varnothing$ (equivalently, $\mathfrak{F}(0, \Delta) \subset \mathfrak{U}(0)$ ).

(2) If $X$ is c-realcompact, then $X$ is a-realcompact.

Proof. (1) If there are $\mathscr{F}^{p}$ with $C I P$ and $\mathscr{U}^{p}$ without $C I P$ for $p \in \mathfrak{F}(0, \triangle) \cap \mathfrak{H}(0, \triangle)$, then cl $\mathscr{U}^{p} \subset \mathscr{F}^{p}$ because $X$ is $(v)$-almost normal, a contradiction.

(2) Since $X$ is c-realcompact, $\mathfrak{u}(0)=\varnothing$, so $\mathfrak{F}(0, \Delta)=\varnothing$ by (1). Thus $\mathfrak{F}(0) \cup \mathfrak{F}(0, \triangle)=\varnothing$ by $2.1(1)$ and hence $X$ is $a$-realcompact.

THEOREM 3.3. (1) $X$ is realcompact iff $X$ is (v)-almost normal and there is a cp $\mathscr{F}^{p}$ without CIP for every $p \in X^{*}$.

( $\left.1^{\prime}\right)$ An almost normal space $X$ is realcompact iff there is a cp $\mathscr{F}^{p}$ without CIP for every $p \in X^{*}$.

(2) A countably paracompact space $X$ is realcompact iff $X$ is a-realcompact and (v)-almost normal.

(2') An almost normal and countably paracompact space $X$ is realcompact iff $X$ is a-realcompact.

(3) $X$ is realcompact iff $X$ is an a-realcompact space with $Z C$.

(4) $X$ is c-realcompact iff there is a wcp $\mathscr{F}^{p}$ without CIP for every $p \in X^{*}$.

(5) An a-realcompact space $X$ is c-realcompact iff $X$ has $(\alpha)$.

(6) An a-realcompact space $X$ is almost realcompact iff $X$ has WOPC. 
(7) If a wa-realcompact space $X$ has (sa), then $X$ is crealcompact.

(8) A normal c-realcompact space is realcompact iff $X$ has OPO.

Proof. $(1) \Rightarrow$ Evident. $\Longleftarrow$. By the assumption, we have $X^{*}=$ $\mathfrak{F}(\triangle) \cup \mathfrak{F}(0, \triangle)$. Let $p \in \cup X-X$ and take a $c p \mathscr{F}^{p}$ without $C I P$. Then there is $\left\{F_{n} \in \mathscr{F}^{p}\right\} \downarrow \varnothing$ and $\left\{U_{n}\right\}_{\text {open }} \downarrow$ such that $F_{n} \subset U_{n}$ and $\cap \operatorname{cl} U_{n}=\varnothing$. We may assume that $X-\operatorname{cl} U_{n} \neq \varnothing, n \in N$. Since $X$ is (v)-almost normal and $p \in \operatorname{cl}_{\beta X} F_{n}$, we have $p \notin \mathrm{cl}_{\beta X}\left(X-\operatorname{cl} U_{n}\right)$. Thus there is $f_{n} \in C(\beta X)$ such that $p \in Z\left(f_{n}\right) \subset \operatorname{cl}_{\beta X} U_{n}$. Then $f=\sum\left(1 / 2^{n}\right) f_{n} \in$ $C(\beta X), p \in Z(f)$ and $Z(f) \cap X=\varnothing$. This is a contradiction because $p \in \cup X-X$, so $\cup X=X$.

(1') From (1).(2) From 1.7(3) and (1). (2') From (2).(3) $\Rightarrow$ Obvious. $\Longleftarrow$ From 1.4(3) and 2.3(8), (4) From 1.1(3), 1.5 and 2.1(2).

(5) If $X$ has $(\alpha)$, then $\mathfrak{H}(0) \cap \mathfrak{F}(\triangle)=\varnothing$ by $2.3(1)$. Since $X$ is $a$-realcompact, we have $\mathfrak{\Im}(0) \cup \mathfrak{F}(0, \Delta)=\varnothing$, and hence $\mathfrak{H}(0)=\varnothing$, so $X$ is $c$-realcompact. The converse follows from the diagram of 2.3.

$(6) \Rightarrow$ From the diagram of 2.3. $\Longleftarrow$ From $1.4(1), \mathfrak{F}(0, \triangle) \cup \mathfrak{F}(0)=$ $\varnothing, 2.3(6)$ and $W O P C$.

( 7 ) Since $X$ has $(s \alpha), \mathfrak{F}(0)=\mathfrak{u}(0)$ by 2.3(2). On the other hand $\mathfrak{F}(0)=\varnothing$ by $w a$-realcompactness, so $\mathfrak{H}(0)=\varnothing$, and hence $X$ is $c$ realcompact.

$(8) \Rightarrow$ Obvious. $\Longleftarrow$. Since $X$ is normal and has $O P O, X$ is weak$c b^{*}$ by 3.1(2). Thus $v X-X=\mathfrak{H}(0)$. On the other hand, $X$ being $c$-realcompact. We have $\mathfrak{u}(0)=\varnothing$ by $1.4(2)$, and hence $X$ is realcompact.

3.4. In the following, we have $(1) \Rightarrow(2) \Rightarrow(3) \Rightarrow(4) \Rightarrow(5) \Leftrightarrow(6)$.

(1) $X$ is realcompact.

(2) $X$ has (s $\beta$ ) and no $F$ has CIP.

(3) $X$ is an almost realcompact space with $(s \beta)$.

(4) $X$ is almost realcompact.

(5) $X$ has (so) and no F has CIP.

(6) $X$ is an a-realcompact space with (s $\alpha$ ) (and hence $X$ is also c-realcompact by $3.3(5))$.

Proof. $\quad(1) \Rightarrow(2),(3) \Rightarrow(4)$ and $(5) \Rightarrow(6)$ are evident. $\quad(4) \Rightarrow(5)$. From 1.4(1), 2.1(1) and $v X-X=\mathfrak{u}(0)$.

$(2) \Rightarrow(3)$ Suppose that $\mathscr{C}=\mathscr{U}^{p}$ has $C I P, p \in \cup X-X$. Let $\mathscr{F} \in$ $\mathfrak{F}(\mathscr{C})$. By the assumption, $\mathscr{F}$ is $c p$ and does not have CIP. There are $\left\{F_{n} \in \mathscr{F}\right\} \downarrow \varnothing$ and $\left\{U_{n}\right\}_{\text {open }} \downarrow$ with $F_{n} \subset U_{n}$ and $\cap \mathrm{cl} U_{n}=\varnothing$. This implies $U_{n} \in \mathscr{C}$ and $\mathscr{U}$ does not have $C I P$, a contradiction.

$(6) \Longrightarrow(5) \quad$ Take $\mathscr{F}=\mathscr{F}^{p}, p \in \cup X-X$. Since $v X-X=\mathfrak{F}(v, \triangle)$, $\mathscr{F}$ does not have $C I P$, so $\mathscr{F}$ is $w c p$ by $(s \alpha)$. 
3.5. In [13], the following theorem was communicated to $\mathrm{P}$. Simon by Z. Frolik:

Theorem F. If $X$ is a normal a-realcompact space, then the realcompactness of $X$ is equivalent to the following condition:

$(S Z C)$ If $\mathscr{F}$ does not have CIP, neither has $\mathscr{F} \cap Z(X)$.

Since $S Z C$ is equivalent to the condition: if $\mathscr{F} \cap Z(X)$ has $C I P$, so has $F$. We have $S Z C \Rightarrow Z C$, and hence the normality in Theorem $F$ is superfluous by $2.3(9)$ and $3.3(3)$.

\subsection{Simon proved the following [13].}

THEOREM S. There is a $\mathscr{\varkappa}^{p}$ with CIP in $X$ such that no $\mathscr{F} \supset$ OP $^{p}$ has CIP where $X$ is the Dowker space in 4.1 belows.

Relating this theorem, we consider the following conditions:

(a) If $\mathscr{Y}^{p}$ has CIP, then no $\mathscr{F}^{p}$ has CIP.

(b) If $\mathscr{Y}^{p}$ has CIP, then there is $\mathscr{F}^{p}$ without CIP.

Then Theorem $\mathrm{S}$ is a direct consequence of the fact that $a$-realcompactness is equivalent to (a). For since $\mathscr{F}^{p}$ has CIP iff $p \in \cup X-X$, it is easy to see that (a) is equivalent to "no $\mathscr{F}^{p}$ has $C I P$ for each $p \in \cup X-X$ " equivalently to " $X-X=\mathfrak{F}(\nu, \triangle)$, i.e., $X$ is $a$-realcompact". Similarly we have that (b) is equivalent to the wa-realcompactness of $X$.

\section{Examples.}

Dowker space 4.1. Let $X$ be the Dowker space, constructed by M. E. Rudin [12], which is normal but not countably paracompact. $X$ is, moreover, weak-cb [6] and $a$-realcompact [13] but not $c$-realcompact [5]. Since $a$-realcompactness $\Leftrightarrow v X-X=\mathfrak{F}(\nu, \triangle), c$-realcompactness $\Leftrightarrow \mathfrak{U}(0)=\varnothing$, normality $\Rightarrow \mathfrak{F}(0, \triangle)=\varnothing$ and weak-cb*ness $\Leftrightarrow v X-X=\mathfrak{H}(0)$, we have $v X-X=\mathfrak{F}(v, \triangle)=\mathfrak{H}(0)$. This shows that $X$ is not $c b^{*}$ and hence $X$ has neither $W O P C$ nor $Z C$ by $2.3(7)$ and 3.1(4) respectively. It is obvious that $X$ does not have $(\alpha)$ by $2.3(1)$.

4.2. Let $X$ be the countably paracompact space, constructed by Mack and Johnson [9] (or see, [11]) is $c$-realcompact [14] and $v X=$ $X \cup\{p\}$ but $X$ is not weak-cb* [8]. But $X$ is neither almost realcompact [14] nor $a$-realcompact [5]. Thus $v X-X=\widetilde{F}(0, \triangle)=$ $\mathfrak{u}(0, \triangle)=\{p\}$. 
Tychonoff Plank 4.3. Let $T=[0, \Omega] \times[0, \omega]-\{p\}$ where $p=$ $(\Omega, \omega) . \quad T$ is pseudocompact but not countably compact and $\beta T=$ $v T=[0, \Omega] \times[0, \omega]$. Since a pseudocompact space is weak-c $b^{*}, \mathfrak{u}(0) \neq$ $\varnothing$. It is easy to see that $\mathfrak{H}(0)=\mathfrak{F}(0, \triangle)=\{p\}$ and $T$ is not $c b^{*}$ [8] and moreover, $T$ has $O P C$ but does not have $C P C$. Let $\mathscr{F}$ be a closed ultrafilter containing the right edge. Obviously $\mathscr{F}$ does not have $C I P$ and $Z(X) \cap \mathscr{F} \neq \mathscr{Z}^{p}$ which shows that $X$ does not have $Z C$.

4.4. Let $X=[0, \Omega]^{2}-\{p\}$ where $p=(\Omega, \Omega)$. Then $v X=[0, \Omega]^{2}$. It is easy to see that $\mathfrak{F}(0)=\mathfrak{u}(0)=\{p\}$.

\section{REFERENCES}

1. N. Dykes, Generalizations of realcompact spaces, Pacific J. Math., 33 (1970), 571-581.

2. Z. Frolik, On almost realcompact spaces, Bull. Acad. Polon Sci., 9 (1961), 247-250.

3. L. Gillman and M. Jerison, Rings of Continuous Functions, Princeton, 1960.

4. K. Hardy and R. G. Woods, On c-realcompact spaces and locally bounded normal functions, Pacific J. Math., 43 (1972), 647-656.

5. K. Hardy, Notes on two generalizations of almost realcompact spaces, Math. Centrum, ZW., (1975), 57-75.

6. K. Hardy and I. Juhasz, Normality and the weak-cb property, Pacific J. Math., 64 (1976), 167-172.

7. F. Ishikawa, On countably paracompact spaces, Proc. Japan Acad., 31 (1955), 686-687.

8. T. Ishiwata, $d_{-}, d^{*}$-maps and $c b^{*}$-spaces, Bull. Tokyo Gakugei Univ., Ser. IV, 29 (1977), 19-52.

9. J. Mack and D. Johnson, The Dedekind completion of $C(X)$, Pacific J. Math., 20 (1967), 231-243.

10. M. Mandelker, Supports of continuous functions, Trans, Amer. Math. Soc., 156 (1971), 73-83.

11. K. Morita, Some properties of M-spaces, Proc. Japan Acad., 43 (1967), 869-872.

12. M. E. Rudin, A normal space $X$ which $X \times I$ is not normal, Fund. Math., 73 (1971), $179-186$.

13. P. Simon, A note on Rudin's example of Dowker space, Comm. Math. Univ. car., 12 (1971), 825-834.

14. R. G. Woods, A Tychonoff almost realcompactification, Proc. Amer. Math. Soc., 45 (1974), 200-208.

Received October 4, 1979 and in revised form September 3, 1980.

Tokyo Gakugei University

ToKyo 184, JAPAN 



\section{PACIFIC JOURNAL OF MATHEMATICS}

\section{EDITORS}

DONALD BABBITT (Managing Editor)

University of Galifornia

Los Angeles, California 90024

Hugo RossI

University of Utah

Salt Lake City, UT 84112

C. C. MOORE AND ANDREW OGG

University of California

Berkeley, CA 94720

\section{J. DugundjI}

Department of Mathematics University of Southern California

Los Angeles, California 90007

R. Finn AND J. Milgram

Stanford University

Stanford, California 94305

\section{ASSOCIATE EDITORS}

R. ARENS

E. F. BECKENBACH

B. H. NeUmanN

F. WOLF

K. YosHIDA

\section{SUPPORTING INSTITUTIONS}

UNIVERSITY OF ARIZONA

UNIVERSITY OF BRITISH COLUMBIA

CALIFORNIA INSTITUTE OF TECHNOLOGY

UNIVERSITY OF CALIFORNIA

MONTANA STATE UNIVERSITY

UNIVERSITY OF NEVADA, RENO

NEW MEXICO STATE UNIVERSITY

OREGON STATE UNIVERSITY
UNIVERSITY OF OREGON

UNIVERSITY OF SOUTHERN CALIFONIA

STANFORD UNIVERSITY

UNIVERSITY OF HAWAII

UNIVERSITY OF TOKYO

UNIVERSITY OF UTAH

WASHINGTON STATE UNIVERSITY

UNIVERSITY OF WASHINGTON 


\section{Pacific Journal of Mathematics}

\section{Vol. 94, No. 1 \\ May, 1981}

Willy Brandal, Conditions for being an FGC domain $\ldots \ldots \ldots \ldots \ldots \ldots \ldots$

Allan Calder and Frank Williams, Incompressibility of maps and the

homotopy invariance of Čech cohomology $\ldots \ldots \ldots \ldots \ldots \ldots \ldots \ldots \ldots$

Jacques Chaumat, Quelques propriétés du couple d'espaces vectoriels

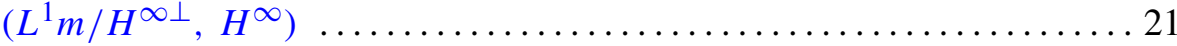

Manfred Droste and Rüdiger Göbel, Products of conjugate permutations . . 47

Jean Esterle, Rates of decrease of sequences of powers in commutative

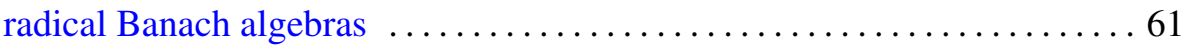

Allan Fryant, Ultraspherical expansions and pseudo analytic functions . . . 83

John Hannah, Homogenization of regular rings of bounded index. II . . . . . 107

Shigeru Haruki, On the theorem of S. Kakutani-M. Nagumo and J. L. Walsh

for the mean value property of harmonic and complex polynomials $\ldots .113$

Hugh M. Hilden, Representations of homology 3-spheres $\ldots \ldots \ldots \ldots \ldots 125$

Craig Huneke, A characterization of locally Macaulay completions . . . . . 131

Takesi Isiwata, Closed ultrafilters and realcompactness ................. 139

Joseph Weston Kitchen, Jr. and David A. Robbins, Tensor products of

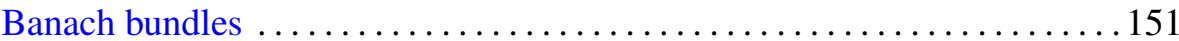

Allan J. Kroopnick, Note on bounded $L^{p}$-solutions of a generalized Liénard

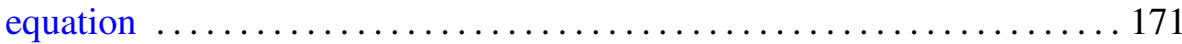

Ajay Kumar and Ajit Kaur Chilana, Spectral synthesis in products and quotients of hypergroups

Charles Livingston, Homology cobordisms of 3-manifolds, knot

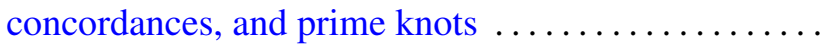

Hans Opolka, Projective representations of finite groups in cyclotomic fields

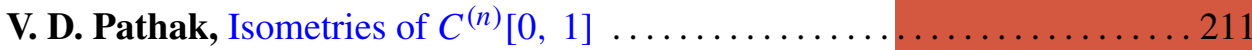

Mark Allan Pinsky, On the spectrum of Cartan-Hadamard manifolds . . . . . 2223

Judith Roitman, The number of automorphisms of an atomic Boolean

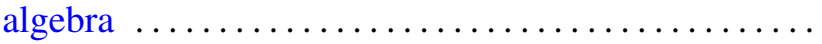

Kai Wang, Locally smooth torus group actions on integral cohomology complex projective spaces 